\title{
6-DoF automatic micropositioning using photometric information
}

\author{
Le Cui ${ }^{1}$, Eric Marchand ${ }^{1}$, Sinan Haliyo ${ }^{2}$ and Stéphane Régnier ${ }^{2}$
}

\begin{abstract}
In this paper, an approach for 6-DoF automatic micropositioning is presented. It involves a closed-loop visual servoing scheme in order to achieve eye-to-hand positioning task in micro-scale. Instead of using classical visual features in the servoing scheme, pure image photometric information from the vision sensor is employed to compute the control law for micropositioning. The approach is validated in simulation as well as experimentally on a parallel positioning stage and a digital microscope at low magnification. Experimental results show the accuracy and efficiency of this control scheme.
\end{abstract}

\section{INTRODUCTION}

The past decade has seen the rapid development of microelectromechanical and microoptoelectromechanical systems (MEMS/MOEMS. These represent a significant potential in the fabrication of smaller components and microstructures hence play an important role in several industrial and biomedical domains, where the integration of these devices would lead to the development of low-cost and highperformance microsystems. Automatic and reliable handling and assembly of these micro-structures is a very active field [1].

Vision is the main spatial sensing technology given the constraints on the microscale, whatever through optical or scanning electron microscopy. Visual servoing is hence an unavoidable tool for automation of the manipulation [2], [3] One of the outstanding issues in this domain is the accuracy in position and orientation of an object in the camera frame [4], [5] and [6]. Most approaches are based on the observation of object features [7], [8]. These are principally geometrical information (eg, corners, edges) or markings on the object surface. By estimating the position and orientation of these features, the pose (position and orientation) of object in the camera frame can be detected. The bottleneck in visual tracking task and object localization is the quality of image from the camera or microscope. The available imaging techniques at the microscale, high magnification optics or SEM, come both with several limitations, including the signal-to-noise ratio, depth of field, refresh rate, contrast...

Recent visual servoing techniques have been proposed to adapt for different issues and imaging conditions. In [9], image gradient information is introduced to control the camera and light source positions. Another similar approach uses the photometric information [10], [11] as a visual feature in control law. In this case, only the image intensity

\footnotetext{
${ }^{1}$ Le Cui and Eric Marchand are with Université Rennes 1, Lagadic team, IRISA, Rennes, France le.cuiduniv-rennesl.fr, eric.marchandeirisa.fr

${ }^{2}$ Sinan Haliyo and Stéphane Régnier are with ISIR, Université Pierre et Marie Curie, Paris, France sinan.haliyo@upmc.fr, stephane.regnierdupmc.fr
}

is needed, the visual tracking process, including feature extraction and motion prediction is no longer necessary. [12] has proved that this approach can be used efficiently in micro-positioning with in 3-DoF (translation in $x$ and $y$ axis, and rotation around $z$ axis). However, in micromanipulation and microassembly, an accurate and reliable control within 6-Dof is expected.

This study addresses a control scheme for visual servoing with photometric information for the micropositioning task in 6 DoF ( 3 translations and 3 rotations). The final purpose is to employ this approach on a scanning electron microscope (SEM) at high accuracy. In this manuscript, the initial validation on a 6 axis parallel-kinematics positioning stage is presented, using first a simulated environment mimicking the visual output of a SEM, then experimentally through a digital microscope.

The manuscript is organized as follows: The classic visual servoing approach and control laws are recalled in Section II A. Section II B describes the camera projection model for the visual servoing. The principle of photometric information as a visual feature is recalled in Section II C. Section III explains the nonlinear optimization and image processing in the visual servoing procedure. The microassembly workcell as well as simulation and experimental results are shown in Section IV.

\section{Photometric Visual SERVOING}

Visual servoing is a technique in which image data form one or more cameras are used in order to control the motion of a robot [13]. In this technique, two kinds of robotcamera relation are considered: eye-in-hand case, in which the camera is installed in the end-effector, and alternatively, the eye-to-hand case, where the camera is fixed and look toward the end-effector. In microrobotics, the eye-to-hand case is generally considered since the sensor (microscope) is motionless.

\section{A. Control law}

The goal of visual servoing is to minimize the error e between the current visual feature $\mathbf{s}(\mathbf{q})$ and the desired one $\mathbf{s}^{*}$.

$$
\mathbf{e}(\mathbf{q})=\mathbf{s}(\mathbf{q})-\mathbf{s}^{*}
$$

The relation between the time deviative $\dot{\mathbf{s}}=\frac{\partial \mathbf{s}}{\partial t}$ and the robot joint velocity $\dot{\mathbf{q}}$ is given by:

$$
\dot{\mathbf{s}}=\mathbf{J}_{s} \dot{\mathbf{q}}
$$

where $\mathbf{J}_{s}$ represents the visual feature Jacobian. 
If we specify an exponential decrease of the error $\dot{\mathbf{e}}=$ $-\lambda \mathbf{e}$, with (1) and (2), the control law can be expressed by:

$$
\dot{\mathbf{q}}=-\lambda \mathbf{J}_{s}^{+} \mathbf{e}
$$

where $\lambda$ is the proportional coefficient and $\mathbf{J}_{s}^{+}$is the pseudoinverse of $\mathbf{J}_{s}$.

Considering the eye-to-hand visual servoing context, the Jacobian $\mathbf{J}_{s}$ can be expressed as:

$$
\mathbf{J}_{s}=-\mathbf{L}_{s}^{c} \mathbf{V}_{F}^{F} \mathbf{J}_{n}(\mathbf{q})
$$

where $\mathbf{L}_{s}$ represents the interaction matrix, which links the relative camera instantaneous velocity $\mathbf{v}$ and the feature motion $\dot{\mathbf{s}},{ }^{c} \mathbf{V}_{F}$ is the motion transform matrix which transforms velocity expressed in camera reference frame onto the robot frame, ${ }^{F} \mathbf{J}_{n}(\mathbf{q})$ is the robot Jacobian in the robot reference frame.

\section{B. Camera projection model}

In microrobotics, the camera projection model depends on the sensor and the image formation. A projection model that could be considered is perspective projection, in which objects are projected towards a point referred to as the center of projection. Alternatively, the parallel projection, in which the projection rays and the image plane are perpendicular, corresponds to a perspective projection with an infinite focal length. It should be considered only at high magnification[14],[15]. In this paper, the experiments are performed at low magnification, only perspective projection model has been employed.

Let ${ }^{c} \mathbf{X}=\left({ }^{c} X,{ }^{c} Y,{ }^{c} Z\right)$ be the coordinates of a point on the observed object expressed in the sensor frame. $\boldsymbol{x}=(x, y)$ is the coordinates of its projection in the image plane expressed, we have

$$
\left\{\begin{array}{l}
x=\frac{{ }^{c} X}{{ }^{c} Z} \\
y=\frac{{ }^{c} Y}{{ }^{c} Z}
\end{array}\right.
$$

The actual image coordinates expressed in pixel $\boldsymbol{x}_{p}=(u, v)$ on the image plane and given by

$$
\left\{\begin{array}{l}
u=u_{0}+p_{x} x \\
v=v_{0}+p_{y} y
\end{array}\right.
$$

where $p_{x}$ and $p_{y}$ represent the pixel/meter ratio and $u_{0}, v_{0}$ the principal point coordinates in the image plane. According to (5) and (6), the general expression is:

$$
\left[\begin{array}{l}
u \\
v \\
1
\end{array}\right]=\left[\begin{array}{ccc}
p_{x} & 0 & u_{0} \\
0 & p_{y} & v_{0} \\
0 & 0 & 1
\end{array}\right]\left[\begin{array}{cccc}
1 & 0 & 0 & 0 \\
0 & 1 & 0 & 0 \\
0 & 0 & 1 & 0
\end{array}\right]\left[\begin{array}{c}
{ }^{c} X \\
{ }^{c} Y \\
{ }^{c} Z \\
1
\end{array}\right]
$$

In visual servoing procedure, the interaction matrix is calculated from this projection model.

\section{Photometric information as a visual feature}

In visual servoing, one or more feature information, such as geometric measurements (ex, position and orientation of interesting points) or direct image information including image gradient [16], [9] and image entropy [17] can be extracted as a visual feature. In this paper, we consider the photometric information from the pure image as the visual feature s. The intensity $\mathbf{I}$ of all the pixels is used. In this case, we define the cost function:

$$
\mathbf{e}(\mathbf{q})=\mathbf{I}(\mathbf{q})-\mathbf{I}^{*}
$$

For a pixel $\mathbf{x}=(x, y)$, the time deviation of $\mathbf{x}$ can be expressed by

$$
\dot{\mathbf{x}}=\mathbf{L}_{\mathbf{x}} \mathbf{v}
$$

where $\mathbf{v}=(v, w)$ contains the relative camera instantaneous linear velocity $v$ and angular velocity $w, \mathbf{L}_{\mathbf{x}}$ is the interaction matrix,

$$
\mathbf{L}_{\mathbf{x}}=\left[\begin{array}{cccccc}
-\frac{1}{Z} & 0 & \frac{x}{Z} & x y & -\left(1+x^{2}\right) & y \\
0 & -\frac{1}{Z} & \frac{y}{Z} & 1+y^{2} & -x y & -x
\end{array}\right] .
$$

Let $I(\mathbf{x}, t)$ be the intensity of the pixel $\mathbf{x}$ at time $t$, then

$$
\nabla I=\left[\begin{array}{cc}
\frac{\partial I}{\partial x} & 0 \\
0 & \frac{\partial I}{\partial y}
\end{array}\right]
$$

the total deviation of the intensity $I(\mathbf{x}, t)$ can be written as

$$
\dot{I}(\mathbf{x}, t)=\nabla I \dot{\mathbf{x}}+\dot{I}
$$

where $\dot{I}=\frac{\partial I}{\partial t}$ represents the time variation of $I$. According to [18] based on the temporal luminance constancy hypothesis, $\dot{I}(\mathbf{x}, t)=0$. In this case,

$$
\dot{I}=-\nabla I \mathbf{L}_{\mathbf{x}} \mathbf{v}=\mathbf{L}_{I} \mathbf{v}
$$

If we consider the entire image, $\mathbf{I}=\left(I_{00}, I_{01}, \cdots, I_{M N}\right)$, where $M, N$ represent the image size, we have

$$
\dot{\mathbf{I}}=\left(\begin{array}{c}
\mathbf{L}_{I_{00}} \\
\vdots \\
\mathbf{L}_{I_{M N}}
\end{array}\right) \mathbf{v}=\mathbf{L}_{\mathbf{I}} \mathbf{v}
$$

where $\dot{\mathbf{I}}$ is the variation of the whole image intensity.

\section{VISUAL SERVOING PROCEDURE}

The visual servoing framework is a closed-loop control system containing a hexapod as the positioning stage, a vision sensor as the image acquisition system and the control system. At the beginning of the procedure, the desired image I $^{*}$ (image of the specimen at a desired position) is acquired. At each iteration of the algorithm, the current image I of the object on the platform is acquired. Robot velocities are computed by the visual servoing scheme in minimizing the image intensity error $\mathbf{e}$ between the desired image $\mathbf{I}^{*}$ and current image $\mathbf{I}(\mathbf{q})$. 


\section{A. Nonlinear optimization}

The visual servoing task can be considered as a nonlinear minimization process. The general idea of minimizing a nonlinear function is to successively update the parameters in order to decreases the value of the cost function $C$ until the error converges.

In micro-scale, several particular numerical problems are induced into the optimization algorithms. In our simulation, a rank deficiency of Jacobian matrix takes place owing to some negligible values of measurement. This leads to the impossibility in computing equation (3). To improve the robustness of algorithm, a Levenberg-Marquardt-like method is considered:

$$
\dot{\mathbf{q}}=-\lambda(\mathbf{H}+\mu \cdot \operatorname{diag}(\mathbf{H}))^{-1} \mathbf{J}_{s}^{\top} \mathbf{e}(\mathbf{q})
$$

where $\mu$ is a coefficient whose typical value ranges from 0.001 to 0.0001 . $\operatorname{diag}(\mathbf{H})$ represents a diagonal matrix of the matrix $\mathbf{H}=\mathbf{J}_{s}^{\top} \mathbf{J}_{s}$.

\section{B. Image processing}

The photometric information may also be sensitive to disturbance in the image. These disturbances come from CCD (charge-coupled device) noise, lighting variation during the experiment and reflection of light on the specimen surface. To achieve good accuracy, these disturbances should be prevented or compensated before computing the image error e. The image processing approach should respect the image original information and be homogeneous for each iteration during the visual servoing procedure.

In order to reduce the CCD noise, we initially compute the average image intensity from a set of images at desired position as the desired image. The images are taken one by one in a time interval $\Delta t$. Let $\mathbf{I}_{i}$ be the acquired image at time $t_{i}$, then $t_{i}=t_{0}+i \Delta t$. The desired image can be expressed as:

$$
\mathbf{I}^{*}=\frac{1}{n}\left(\mathbf{I}_{1}+\mathbf{I}_{2}+\ldots+\mathbf{I}_{n}\right)
$$

where $n$ is the image number. Similar as desired image, every current image is computed by a set of images of current position after the motion of positioning stage in every iteration.

A median filter is simultaneously employed in the procedure for reducing the CCD noise. Let $I_{f}(x, y)$ be the filtered image intensity at pixel $(x, y)$, then

$$
I_{f}(x, y)=\operatorname{med}\left(I\left(x^{\prime}, y^{\prime}\right) \mid\left(x^{\prime}, y^{\prime}\right) \subset W(x, y)\right)
$$

where $W(x, y)$ represents all the pixels in a $w \times w$ window centered at $(x, y)$, med means the median of the gray levels of these pixels.

Depending on the situation, a Gaussian filter can be alternatively introduced for denoising. However, the standard deviation of Gaussian kernel should not be large in order to keep the sharpness of the image. Since both noise and blur induce lesser accuracy, the image filter should be employed cautiously.

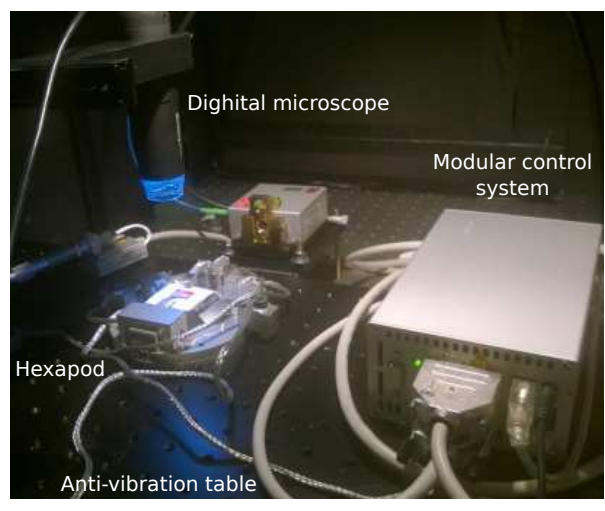

Fig. 1. The micropositioning workcell

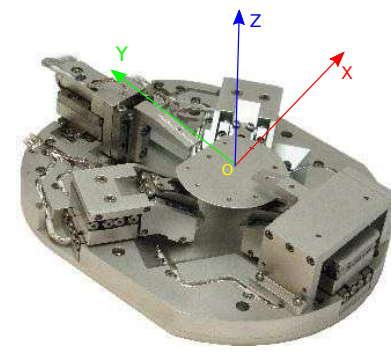

(a)

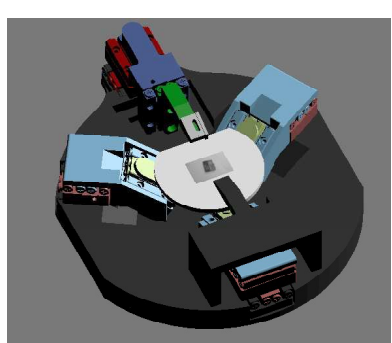

(b)
Fig. 2. (a) Positioning stage and (b) its 3D model

\section{EXPERIMENTAL RESULTS}

\section{A. Experimental setup}

Experiments were conducted with a micropositioning workcell shown in Fig. 1. This workcell is installed on an anti-vibration table. It contains a 6-DoF positioning stage SmarPod 70.42-S-HV made by SmarAct including its positioner SLC 17.20-S-HV as well as its modular control system and a digital microscope Veho VMS-004D towards the topplate of positioning stage. It provides $20 \times$ or $400 \times$ magnification. Our experiments were realized at a magnification of $20 \times$.

TABLE I

Positioning STAGE SPECIFICATIONS

\begin{tabular}{c|c|c}
\hline & Travel range & Closed-loop resolution \\
\hline $\mathrm{X}$ & $+/-6 \mathrm{~mm}$ & $1 \mathrm{~nm}$ \\
$\mathrm{Y}$ & $+/-6 \mathrm{~mm}$ & $1 \mathrm{~nm}$ \\
$\mathrm{Z}$ & $+/-3 \mathrm{~mm}$ & $1 \mathrm{~nm}$ \\
\hline$\theta_{X}$ & $+/-10^{\circ}$ & $1 \mu \mathrm{rad}$ \\
$\theta_{Y}$ & $+/-10^{\circ}$ & $1 \mu \mathrm{rad}$ \\
$\theta_{Z}$ & $+/-20^{\circ}$ & $1 \mu \mathrm{rad}$ \\
\hline
\end{tabular}

The SmarPod positioning stage is a parallel robot (hexapod) that provides three positioners that support the top-plate. By the motion of positioners, the top-plate can be moved in three directions and rotated around three axes. The hexapod and the reference frame are shown in Fig. 2(a). Table I describes its specifications.

The experimental framework is shown in Fig. 3. The proposed visual servoing procedure has been implemented with 


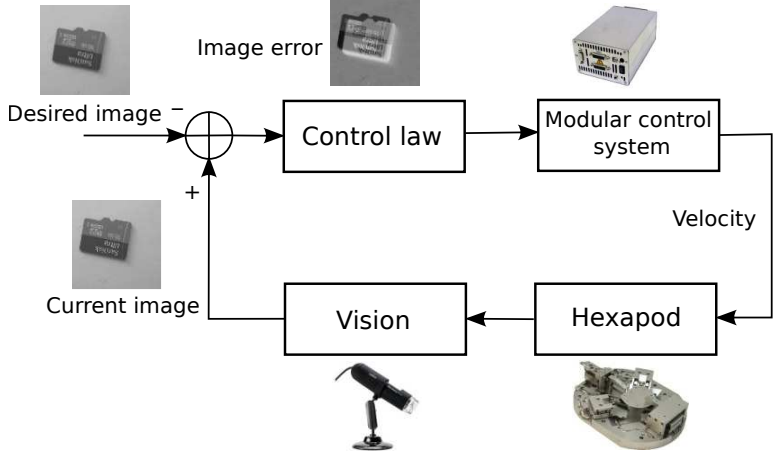

Fig. 3. Experimental framework
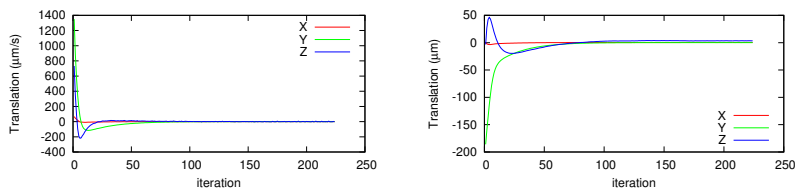

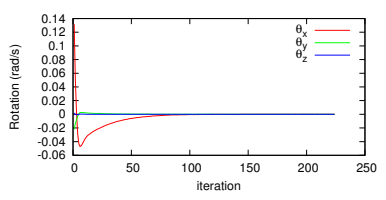

(a)

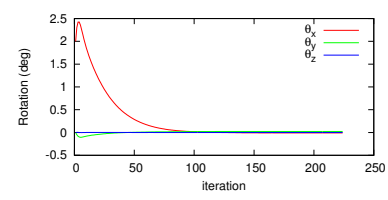

(b)
Fig. 4. Simulation results, $2^{\circ}$ rotation around $x$ axis as the initial pose (a) Evolution of joint velocity (in $\mu \mathrm{m} / \mathrm{s}$ and $\mathrm{rad} / \mathrm{s}$ ). (b) Evolution of object pose error (in $\mu \mathrm{m} / \mathrm{s}$ and degree)

the ViSP library [19]. Visual servoing control calculation and image processing were performed on a laptop running Linux. The connection of the PC with modular control system and with digital microscope is established by USB port.

\section{B. Simulation results}

To validate the visual servoing process, simulations were conducted by using a full 3D model of the positioning stage (see Fig. 2(b)), based on software Blender. The procedure of visual servoing as well as the calibration is as similar as that in the experiment. An image of the specimen acquired by digital camera is fixed on the top-plate of the positioning stage to perform a similar condition of experiment. The acquired image size is $512 \times 512$ pixels.

Initially, we test the behavior and performance of the proposed algorithm. A simple rotation of $2^{\circ}$ around $x$ axis is sent to positioning stage as a initial pose. Fig. 4 shows the result of simulation. The algorithm converges quickly and the accuracy reaches $0.26 \mu \mathrm{m}, 0.07 \mu \mathrm{m}$ and $3.01 \mu \mathrm{m}$ in translation on $x, y, z$ axes; $0.003^{\circ}, 0.020^{\circ}$, and $0.0001^{\circ}$ in rotation around $x, y, z$ axes, respectively. The results show that in positioning at micro/nano-scale, the position in $z$ axis is more difficult to control in visual servoing than the other two axes owing to the less observability of motion on $z$ axis under the microscope.

Another positioning simulation was realized by setting an initial pose different from the desired one for all the degrees of freedom. The initial pose of the positioning stage is set to
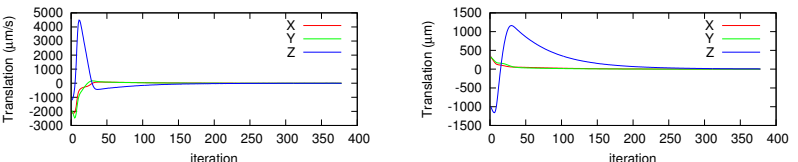

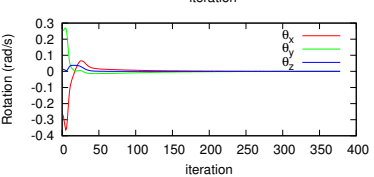

(a)

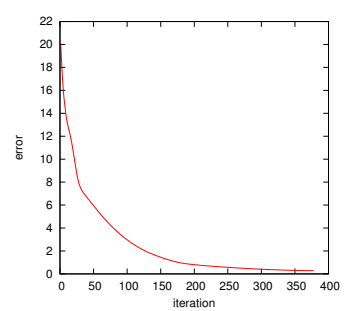

(c)

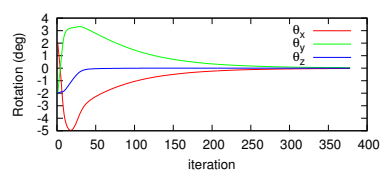

(b)

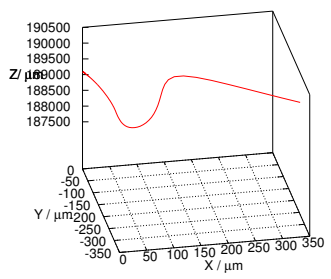

(d) be $500 \mu \mathrm{m}$ both in $x$ and $y$ axes, $-1 \mathrm{~mm}$ in $z$ axis; $2^{\circ}$ around $x$ axis, $-2^{\circ}$ both around $y$ and $z$ axes. The repositioning task is achieved in 380 iterations. Fig. IV-B illustrates the result. To demonstrate the performance, the image intensity error per pixel between the current image and the desired image, which corresponds to equation (8), is computed at each iteration (see Fig. 5(c)). Its value attains 0.2 at the end of procedure. Fig. 5(d) shows the object trajectory in camera reference frame. The accuracy of the object pose error in the end reaches $0.46 \mu \mathrm{m}, 0.24 \mu \mathrm{m}$ and $5.19 \mu \mathrm{m}$ in translation on $x, y, z$ axes; $0.012^{\circ}, 0.030^{\circ}$ and $0.0003^{\circ}$ in rotation around $x, y, z$ axes, respectively.

We experimentally find that the CCD noise in our digital microscope is so large that it can not be easily eliminated. To simulate this, an additional dynamic Gaussian noise is introduced into each acquired image during the simulation. Consequently, we find that the introduction of dynamic noise disturbs the computation of velocity, which may lead to divergence in simulation. In order to compare the performance of algorithm with and without noise, the mean of the Gaussian noise is set to be null and the standard deviation $\sigma=2$. Results are shown in Fig. 5. With respect to Fig. IV$\mathrm{B}$, it has been found that the image error per pixel decreases to 2.9 finally due to image noise and the desired position could be reached with less accuracy as can be expected when velocity converges. This means that to achieve a better accuracy by the photometric visual servoing approach, a denoising procedure such as that we discussed in Section III $\mathrm{B}$ is required.

\section{Experimental results on the actual Smarpod}

In the experiment with micropositioning workcell, we firstly observe the CCD noise from the digital microscope. An experiment has been accomplished in which we keep the positioning stage motionless. In order to observe the CCD noise with respect to time, 200 images are taken with a 

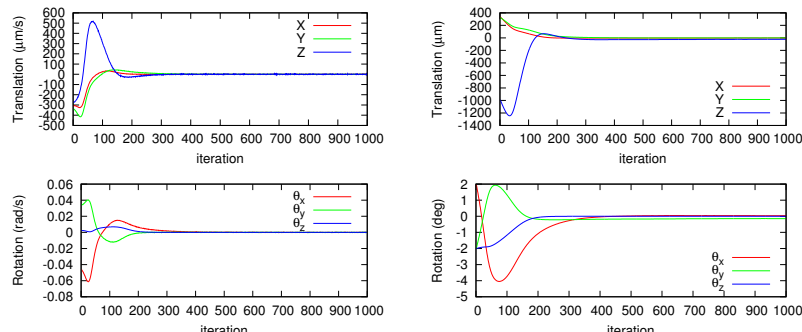

(e)

(f)
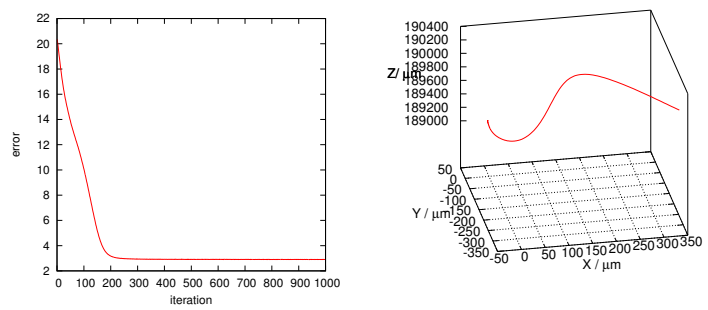

(g)

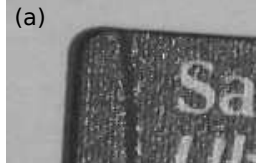

(b)

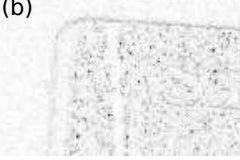

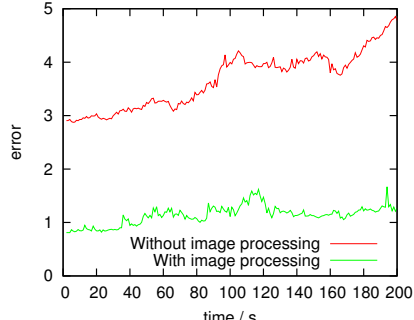

(c)

Fig. 6. (a) Zoom of the an image from digital camera. (b) noise map of (a). (c) Image error per pixel with respect to time
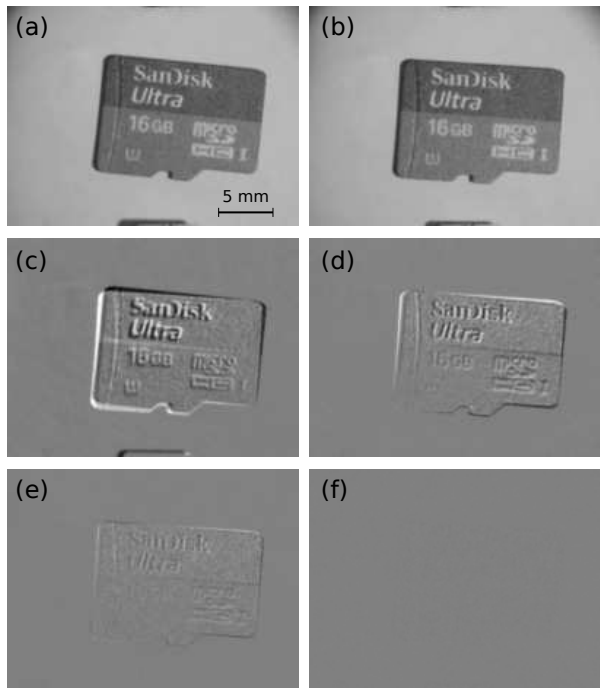

Fig. 7. (a) Initial image, (b) desired image, (c) to (e) show image error $\mathbf{e}(\mathbf{q})$ at 1 st, 30th, $46 t h$ iteration, (f) image error in the end

image which contains a corner of the specimen, In its noise map (see Fig. 6(b)), a pixel is darker when the variation of image intensity is greater and is brighter when a lesser variation of image intensity is found. It can be seen that the noise appears mostly in the edges and the surface of the specimen. To reduce the influence of CCD noise, the image processing approach described in Section III B is applied. The effect of this process is shown in Fig. 6(c), the image error per pixel is diminished to about 1 instead of 3 without image processing.

Furthermore, we find the lighting variation during the positioning procedure could introduce disturbance to computation of control law. When the lighting around the positioning stage changes, the totality of image intensity varies. That leads to a great increase in velocity. The light variation in our experiments is caused mainly by exterior lighting variation. The LEDs lighting from the digital microscope is also variable according to environment lighting. To reduce the indirect lighting variation caused by exterior lighting, black cardboards are installed around the workcell.

The experiment of positioning is then accomplished with the micropositioning workcell. The specimen measures 15 $\mathrm{mm} \times 11 \mathrm{~mm}$, with $0.8 \mathrm{~mm}$ thickness. The initial pose of positioning stage is set to be $500 \mu \mathrm{m}$ both in $x$ and $y$ axes, -1 $\mathrm{mm}$ in $z$ axis; $2^{\circ}$ around $x$ axis, $-2^{\circ}$ both around $y$ and $z$ axes. The initial image and desired image after image processing is shown in Fig. 7(a) and Fig. 7(b), respectively. Fig. 7(c) to

Fig. 7(d) shows the evolution of image error $\mathbf{e}(\mathbf{q})=\mathbf{I}(\mathbf{q})-\mathbf{I}^{*}$ until the end of visual servoing procedure. As a consequence of minimization, the error image is almost null.

The experimental results are shown in Fig. 8. The image error per pixel decreases to 0.9 when the velocity converges. The accuracy of object pose error reaches $0.67 \mu \mathrm{m}, 0.64 \mu \mathrm{m}$ and $3.12 \mu \mathrm{m}$ in translation on $x, y, z$ axes; $0.022^{\circ}, 0.028^{\circ}$ and $0.002^{\circ}$ in rotation around $x, y, z$ axes, respectively. If we regard the accuracy in each axis of both experimental and simulation results, it is clear that the accuracy of these 3 DoF is worse than the others. Practically, in micro-nano scale, the translation on $z$ is difficult to observe due to the microscope projection model. As many microscopes provide a long focal length, a great translation on $z$ effects tiny variation on observed image. The control of rotation around $x$ and $y$ axes is another challenge in microrobotics. When the focal length of microscope is long compared with the motion in $x-y$ plane, the tiny translations on $x$ axis and rotations around $y$ axis are very difficult to be distinguished by image information, and vice versa.

\section{CONCLUSIONS}

We have addressed in this paper a 6-DoF automatic micropositioning approach using photometric information. In 

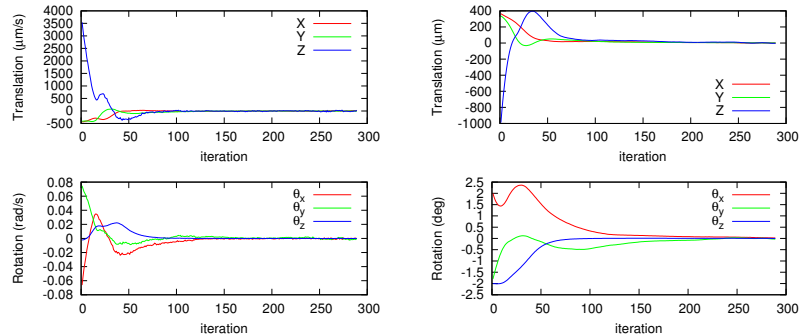

(a)

(b)

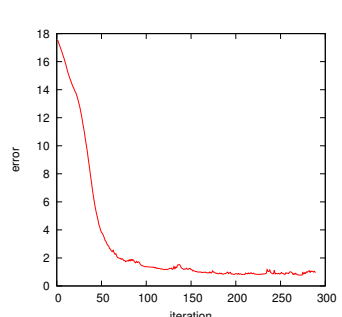

(c)

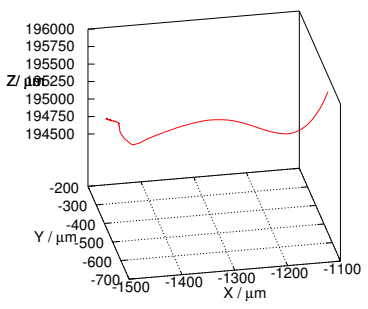

(d)
Fig. 8. Experimental results: (a) Evolution of joint velocity (in $\mu \mathrm{m} / \mathrm{s}$ and $\mathrm{rad} / \mathrm{s}$ ). (b) Evolution of object pose error (in $\mu \mathrm{m} / \mathrm{s}$ and degree). (c) Evolution of image error per pixel. (d) Object trajectory in camera reference frame

this method, pure image intensity is used as a visual feature in visual servoing. A closed-loop control framework of a visual sevoing task has been designed. The approach has been validated by a workcell containing a digital microscope and a 6-DoF hexapod. Even with large differences between the initial pose and desired pose $(0.5 \mathrm{~mm}$ and $1 \mathrm{~mm}$ in translation and $2^{\circ}$ in rotation), accurate results were obtained (below $1 \mu \mathrm{m}$ in translation on $x$ and $y$ axes and $3 \mu \mathrm{m}$ in translation on $z$ axis; below $0.03^{\circ}$ in rotation around $x$ and $y$ axes and $0.002^{\circ}$ in rotation around $z$ axis). As an advantage, very little information (only image intensity) is required in this approach. As the varying illumination leads to inaccurate motion and risk of diverge, image processing should be applied during the positioning stage. Since this visual servoing scheme has been validated under a digital microscope, future work could be to employ it under a scanning electron microscope. In order to achieve this, camera projection model should be changed into parallel projection for a high magnification. The cost function and control law should be modified to reach the high accuracy and to keep the motion in $z$ axis controllable.

\section{ACKNOWLEDGMENT}

The authors would like to thank Jean Ochin Abrahamians and Mokrane Boudaoud for their assistance during the experiments at ISIR, Université Pierre et Marie Curie. The positioning stage is supported by French Robotex platform. This work was realized in the context of the French ANR P2N Nanorobust project (ANR-11-NANO-0006).

\section{REFERENCES}

[1] S. Régnier and N. Chaillet, Eds., Microrobotics for Micromanipulation. Wiley-ISTE, 2010.
[2] C. Pawashe and M. Sitti, "Two-dimensional vision-based autonomous microparticle manipulation using a nanoprobe," Journal of $\mathrm{Mi}$ cromechatronics, vol. 3, no. 4, pp. 285-306, July 2006.

[3] S. Fatikow, C. Dahmen, T. Wortmann, and R. Tunnell, "Visual feedback methods for nanohandling automation," I. J. Information Acquisition, vol. 6, no. 3, pp. 159-169, 2009.

[4] S. Ralis, B. Vikramaditya, and B. Nelson, "Micropositioning of a weakly calibrated microassembly system using coarse-to-fine visual servoing strategies," Electronics Packaging Manufacturing, IEEE Transactions on, vol. 23, no. 2, pp. 123-131, Apr 2000.

[5] S. Devasia, E. Eleftheriou, and S. Moheimani, "A survey of control issues in nanopositioning," Control Systems Technology, IEEE Transactions on, vol. 15, no. 5, pp. 802-823, 2007.

[6] N. Ouarti, B. Sauvet, S. Haliyo, and S. Rgnier, "Robposit, a robust pose estimator for operator controlled nanomanipulation," Journal of Micro-Bio Robotics, vol. 8, no. 2, pp. 73-82, 2013.

[7] Y. Sun, M. Greminger, D. Potasek, and B. Nelson, "A visually servoed mems manipulator," in Experimental Robotics VIII. Springer, 2003, pp. 255-264.

[8] N. Ogawa, H. Oku, K. Hashimoto, and M. Ishikawa, "Microrobotic visual control of motile cells using high-speed tracking system," Robotics, IEEE Transactions on, vol. 21, no. 4, pp. 704-712, 2005.

[9] E. Marchand and C. Collewet, "Using image gradient as a visual feature for visual servoing," in IEEE/RSJ Int. Conf. on Intelligent Robots and Systems, IROS'10, Taipei, Taiwan, October 2010.

[10] C. Collewet, E. Marchand, and F. Chaumette, "Visual servoing set free from image processing," in IEEE Int. Conf. on Robotics and Automation, ICRA'08, Pasadena, CA, May 2008, pp. 81-86.

[11] C. Collewet and E. Marchand, "Photometric visual servoing," IEEE Trans. on Robotics, vol. 27, no. 4, pp. 828-834, August 2011.

[12] B. Tamadazte, N. Le-Fort Piat, and E. Marchand, "A direct visual servoing scheme for automatic nanopositioning," IEEE/ASME Transactions on Mechatronics, vol. 17, no. 4, pp. 728-736, 2012.

[13] F. Chaumette and S. Hutchinson, "Visual servo control, Part I: Basic approaches," IEEE Robotics and Automation Magazine, vol. 13, no. 4, pp. 82-90, December 2006.

[14] M. Hemmleb and J. Albertz, "Microtopography-the photogrammetric determination of friction surfaces," International Archives of Photogrammetry and Remote, vol. 33, pp. 56-63, 2000.

[15] P. G. T. Howell, "A theoretical approach to the errors in sem photogrammetry," Scanning, vol. 1, no. 2, pp. 118-124, 1978.

[16] E. Marchand and N. Courty, "Controlling a camera in a virtual environment: Visual servoing in computer animation," The Visual Computer Journal, vol. 18, no. 1, pp. 1-19, Feb. 2002.

[17] A. Dame and E. Marchand, "Mutual information-based visual servoing," IEEE Trans. on Robotics, vol. 27, no. 5, pp. 958-969, Oct. 2011.

[18] B. Horn and B. Schunck, "Determining optical flow," Artificial Intelligence, vol. 17, no. 1-3, pp. 185-203, Aug. 1981.

[19] E. Marchand, F. Spindler, and F. Chaumette, "ViSP for visual servoing: a generic software platform with a wide class of robot control skills," IEEE Robotics and Automation Magazine, vol. 12, no. 4, pp. 40-52, Dec. 2005. 